Meta

Journal des traducteurs

Translators' Journal

\title{
Simple cicérone au Levant ou véritable truchement ? L'interprète dans les récits journalistiques anglo-saxons
}

\section{Benoit Léger}

Volume 64, numéro 3, décembre 2019

URI : https://id.erudit.org/iderudit/1070532ar

DOI : https://doi.org/10.7202/1070532ar

Aller au sommaire du numéro

\section{Éditeur(s)}

Les Presses de l’Université de Montréal

ISSN

0026-0452 (imprimé)

1492-1421 (numérique)

Découvrir la revue

Citer cet article

Léger, B. (2019). Simple cicérone au Levant ou véritable truchement ? L'interprète dans les récits journalistiques anglo-saxons. Meta, 64(3), 630-647. https://doi.org/10.7202/1070532ar

\section{Résumé de l'article}

Cet article étudie la perception du rôle du fixeur, soit l'interprète professionnel ou ponctuel qui travaille auprès des journalistes étrangers dans une zone de conflit. Robert Fisk, Fred A. Reed, Hugh Pope et Graeme Smith ont couvert le Proche-Orient, l'Asie centrale ou la Turquie pendant des périodes de tensions ou de conflits en faisant appel à des fixeurs qui leur permettent de décrire la situation dans les récits qu'ils publient par la suite. La manière dont ces journalistes décrivent le rôle, la fonction et même l'apparence de leurs fixeurs diffère cependant grandement : certains les mettent au premier plan, évoquant un collaborateur et même une figure parentale, tandis que d'autres mentionnent à peine le besoin d'un médiateur linguistique, et ce, même s'il est évident qu'ils ne maîtrisent pas la langue du pays. La comparaison de ces descriptions du rôle des médiateurs linguistiques éclaire d'un jour nouveau la perception que l'on a de l'interprétation dans les zones de conflit, la fiction d'une communication instantanée entre les langues et, de manière générale, la reconnaissance due aux fixeurs dans le monde du journalisme. 


\title{
Simple cicérone au Levant ou véritable truchement? L'interprète dans les récits journalistiques anglo-saxons
}

\author{
BENOIT LÉGER \\ Université Concordia, Montréal, Canada \\ benoit.leger@concordia.ca
}

\section{RÉSUMÉ}

Cet article étudie la perception du rôle du fixeur, soit l'interprète professionnel ou ponctuel qui travaille auprès des journalistes étrangers dans une zone de conflit. Robert Fisk, Fred A. Reed, Hugh Pope et Graeme Smith ont couvert le Proche-Orient, l'Asie centrale ou la Turquie pendant des périodes de tensions ou de conflits en faisant appel à des fixeurs qui leur permettent de décrire la situation dans les récits qu'ils publient par la suite. La manière dont ces journalistes décrivent le rôle, la fonction et même l'apparence de leurs fixeurs diffère cependant grandement: certains les mettent au premier plan, évoquant un collaborateur et même une figure parentale, tandis que d'autres mentionnent à peine le besoin d'un médiateur linguistique, et ce, même s'il est évident qu'ils ne maîtrisent pas la langue du pays. La comparaison de ces descriptions du rôle des médiateurs linguistiques éclaire d'un jour nouveau la perception que l'on a de l'interprétation dans les zones de conflit, la fiction d'une communication instantanée entre les langues et, de manière générale, la reconnaissance due aux fixeurs dans le monde du journalisme.

\begin{abstract}
This paper analyzes four visions of the role of the fixer - the amateur or professional interpreter working alongside foreign journalists, mostly in conflict zones. Robert Fisk, Fred A. Reed, Hugh Pope and Graeme Smith have covered the Middle East, Central Asia or Turkey during periods of social unrest, military operations and wars, relying to various extents on the ability of local fixers to convey information in their books. The ways in which these journalists describe the role, function, and even appearance of their fixers differ widely: some insist on the presence of a fixer, described as a collaborator or almost as a mother figure, others barely mention the need for language mediation, even in situations where they obviously do not master the language. Confronting these descriptions of mediation between languages sheds light on how the importance of interpretation in conflict zones is perceived, on the fiction of communication without language borders and, more broadly, on the recognition of the role of fixers within the realm of journalism.
\end{abstract}

\section{RESUMEN}

Este artículo estudia la percepción del papel del fixer, es decir el intérprete profesional u ocasional que trabaja con periodistas extranjeros en una zona de conflicto. Robert Fisk, Fred A. Reed, Hugh Pope et Graeme Smith, cubrieron el Medio Oriente, Asia Central o Turquía durante períodos de tensión social o conflicto armado y recurrieron a fixers que les permitieron entender la situación que describieron en los libros que publicaron luego. La forma en que estos periodistas presentan el papel, la función e incluso la apariencia de sus fixers difiere mucho: algunos les otorgan un papel primordial calificándolos de colaboradores o incluso protectores, mientras que otros, incluso si resulta evidente que no dominan la lengua del país, apenas mencionan la necesidad de un mediador lingüístico. La comparación de las descripciones del papel de los mediadores lingüísticos ofrece 
una nueva perspectiva acerca de la percepción que se tiene de la interpretación en zonas de conflicto, la ficción de una comunicación instantánea entre lenguas o, de manera general, el reconocimiento que se merecen los fixers en el ambiente periodístico.

\section{MOTS-CLÉS/KEYWORDS}

interprétation, fixeurs, Proche-Orient, Asie centrale, journalisme interpreting, fixers, Middle East, Central Asia, journalism interpretación, fixers, Mecio Oriente, Asia Central, periodismo

Le cavalier peut aller où bon lui semble, à sa guise; sa carabine est son garde du corps; il est libre comme l'oiseau. Lorsqu'il sait la langue et connaît les mœurs du pays qu'il traverse, lorsqu'il peut se passer d'interprètes, de lettres de recommandation et de guides protecteurs, un pareil voyage devient une partie de plaisir.

(Vambéry 1886: 373) ${ }^{1}$

\section{Introduction}

Cet article s'intéresse au cas d'un certain type d'interprètes, les fixeurs, qui permettent aux journalistes de produire articles et livres sur les zones de conflit, en particulier à leur absence, tout aussi, sinon plus signifiante, que leur présence textuelle ${ }^{2}$. Lorsque j'ai traduit un premier livre du journaliste Hugh Pope, consacré aux peuples turciques d'Asie centrale, je me suis demandé comment le reporter se débrouillait. Comment Pope arrivait-il, par exemple, à embarquer sur le ferry qui l'amènerait du Turkménistan vers l'Azerbaïdjan? Après une journée éprouvante durant laquelle sa Zighuli soviétique tombe en panne et où son chauffeur doit partir seul en quête de pièces de rechange, le journaliste finit par atteindre le port de Turkmenbashy:

We pulled up in front of the main hotel of the Caspian Sea port of Turkmenbashy. I was on my way to Azerbaijan [...]. When I went down to the docks early in the morning, luck seemed to be on my side. A substantial, new-looking rail ferry was in port. The clerks at the shipping office thought it might sail in the afternoon. I should keep coming back every a [sic] few hours to check, they advised, not quite looking $m e$ in the eye. (Pope 2010: 320; nous soulignous)

À l'occasion d'un échange de courriels, j'ai fait part de mon émerveillement à l'auteur qu'il arrive à négocier l'achat de billets, puis à trouver une place sur le ferry. Pope m’a répondu qu'il était rarement seul, mais que la «fiction» du journalisme exige que l'on omette la présence d'un chauffeur ou d'un fixeur, pour ne pas lasser les lecteurs par la mention continuelle d'un intermédiaire. Le traducteur en moi s'est toujours intéressé à la représentation des traducteurs et interprètes, de même qu'à l'absence de difficultés de communication liées au contact des langues, tant en littérature que dans les essais et les films. J'ai donc cherché à mieux comprendre comment les journalistes décrivent l'intermédiaire qui leur permet de communiquer avec leurs interlocuteurs.

Il ne s'agira pas ici d'analyser le rôle des interprètes et des fixeurs en général (même s'il convient de l'aborder) dans le contexte des conflits au Proche-Orient, mais plutôt la perception qu'en ont, l'image qu'en transmettent quatre reporters anglo-saxons ainsi que l'absence (ou quasi-absence) de représentation de ces interprètes, même lorsqu'il est évident que l'un d'eux est présent, alors qu'il n'est jamais 
mentionné ou à peine décrit. On peut affirmer de manière générale qu'il s'agit d'un tabou, que les lecteurs modernes et occidentaux (dans le cas présent) préfèrent accepter le «contrat de fiction» sous-entendu par Pope, ce qui n'empêche pas de se demander si tous les journalistes sont aussi soucieux de gommer l'image d'un intermédiaire linguistique. Si cet intermédiaire n'est pas qu'un simple accompagnateur, s'il joue un rôle indispensable, on est ensuite en droit de se demander pourquoi il demeure à l'arrière-plan dans la plupart des récits journalistiques. S'agit-il encore une fois de préserver la fiction (orientaliste dans le cas qui nous intéresse) d'une communication instantanée et d'une universalité des échanges?

\section{Le fixeur et son rôle}

Le terme de fixeur s'est imposé dans la presse écrite ces dernières années. L'Office québécois de la langue française (OQLF), fidèle à sa mission d'empêcher l'intrusion de tout anglicisme, se prononce contre cet usage, mais propose tout de même une première définition:

Comment traduire ce que les journalistes appellent un fixeur? Mais d'abord, qu'est-ce qu'un fixeur? C'est cette personne, souvent native du pays, qui accompagne le journaliste étranger dans l'exercice de son métier, qui lui facilite les choses en lui obtenant des documents, en établissant les premiers contacts. C'est aussi celui qui conseille, guide ou met en garde le journaliste qui ne connaît pas le pays et lui sert d'interprète lorsque ce dernier ne parle pas la langue. ${ }^{3}$

L'OQLF suggère donc de traduire un terme déjà courant chez les journalistes et propose des «équivalents» destinés à éviter le barbarisme morphologique:

En français, on parlera de guide, ou de guide-interprète si c'est le cas, ou encore d'accompagnateur. Le terme anglais fixer est à éviter et la forme francisée fixeur, qui commence à s'employer dans la presse francophone, n'est pas recommandée puisqu'on ne peut la rattacher en français à un verbe fixer, de sens analogue, duquel fixeur devrait dériver; l'anglais to fix, au contraire, connait des sens qui correspondent au substantif fixer, soit «préparer, arranger, faire avoir, obtenir». (Voir note 2)

L'Office renchérit en reconnaissant au fixeur des qualités qui, on le verra encore, sont assez souvent soulignées par les reporters:

... on a parfois accolé l'appellation ange gardien à celle de fixer dans la presse francophone. Sans être un synonyme parfait de guide-interprète, cette désignation peut s'y substituer dans un contexte où il est clair que le guide fait preuve d'une grande générosité, d'un dévouement, d'un certain attachement pour le ou la journaliste qu'il accompagne, traits qui ne sont pas nécessairement implicites dans les appellations plus neutres de guide-interprète ou d'accompagnateur. (Voir note 2)

Les équivalents "plus neutres» proposés ici mettent l'interprète en apposition, faisant de son rôle de langagier un aspect parmi d'autres, comme une activité ancillaire de chaperon, pour ainsi dire de simple «gentil accompagnateur». Ajoutons que, compte tenu des risques encourus, la désignation «ange gardien» ou «accompagnateur» n'est pas sans évoquer une croisière ou une colonie de vacances où l'on s'amuse.

En 2005, Le Monde expliquait déjà le sens à donner au néologisme:

Le «fixeur» [...] est «l'arrangeur» en chef, le guide-interprète de l'envoyé spécial. Il a des amis ou des connaissances dans le maximum de milieux sociaux. Le journaliste 
confie au «fixeur» son projet de reportage ou d'interview pour la journée. Il peut suggérer des noms, mais c'est souvent au "fixeur» de dénicher le bon interlocuteur. ${ }^{4}$

Ces définitions rappellent, à l'instar d'Alfred Hermann, que l'interprète est un médiateur entre deux parties dont le rôle ne se limite pas à celui de truchement: « [...] the term designates the human mediator positioned between two parties or values, performing far more diverse activities than simply providing linguistic mediation between parties transacting business» (Apostolou 2009: 3).

\subsection{Le type d'interprétation}

On peut définir le travail du fixeur comme celui d'un interprète de liaison qui traduit les questions et réponses d'interlocuteurs qui ne parlent pas la même langue. Selon l'Ordre des traducteurs, terminologues et interprètes agréés du Québec (OTTIAQ), l'interprète doit posséder «une grande capacité de concentration, une excellente mémoire, une voix agréable, une élocution irréprochable ainsi qu'une bonne résistance physique et mentale», qualités auxquelles s'ajoutent «le sens du détail et de la nuance, une grande culture et de solides connaissances générales». Le type d'interprète ainsi décrit n'est pas précisé; la description du métier est suivie d'une précision sur les interprètes de conférence: "L'interprète de conférence doit être disposé à voyager car son travail l'amène souvent à se déplacer hors du pays $»^{5}$ ainsi que de commentaires généraux, par exemple sur les horaires irréguliers des interprètes de conférence ou judiciaires.

Les fixeurs font pourtant beaucoup plus et, pour ainsi dire, beaucoup moins: au-delà de la "culture générale» et des "connaissances générales», qui ne sont pas nécessairement recherchées par les donneurs d'ouvrage, concentrons-nous sur des caractéristiques qui ne sont pas évoquées par l'OTTIAQ:

Words are omitted, sentences changed, meanings altered, since the simultaneous nature of the process and the speed of spoken discourse make faithfulness and accuracy - two imperatives in written translation - impossible. This manipulation by the interpreter can even reach the extreme point of censorship, whether requested by the organizers, or considered necessary by the interpreter for political, cultural or other reasons. (Apostolou 2009: 8)

Ainsi donc, le fixeur omet, transforme, manipule, ajoute, à la manière des interprètes en milieu communautaire.

Ces fixeurs se recrutent dans toutes les couches de la société: employés des ambassades étrangères, officiers de l'armée, chauffeurs de taxi, anciens combattants, enseignants, journalistes locaux ou encore simples gamins ${ }^{6}$. Ce sont, compte tenu des pays concernés et de la situation politique, pour l'essentiel des hommes.

Le travail des fixeurs a été analysé plus en détail dans le monde anglo-saxon, où ces derniers semblent souvent choisis essentiellement pour leurs compétences linguistiques ou ce que l'on peut en établir, et non pour leurs expériences antérieures ou pour leur formation 7 . Leanza et Boivin (2007) s'intéressent au rapport entre parole et pouvoir, et au maintien de l'ordre établi, à la médiation entre systèmes de sens et, surtout, aux métaphores «instrumentales» (Leanza et Boivin 2007: 3, 4) employées pour décrire le travail de l'interprète et à son "pouvoir d'intervention» (Leanza et Boivin 2007 : 9). Les auteurs donnent quelques exemples de «métaphores» recensées 
par d'autres chercheurs, qui montrent en fait que l'imaginaire est ici limité: «collaborateur", "co-travailleur», "co-thérapeute», mais aussi "machine à traduire", "poteau de téléphone» ou encore "voice box/black box». Leanza et Boivin en concluent que l'interprète, «invisible ou déshumanisé», «est sans pouvoir aussi bien sur l'usager que sur le praticien ou sur la relation» et qu'il n'a pas "la capacité de produire un discours propre ou de transformer celui des interlocuteurs» (Leanza et Boivin 2007: 8).

Bien que Fernández-Ocampo et Wolf (2014) s’intéressent aux clichés photographiques montrant les interprètes, une partie de leur analyse sert ici notre propos. Les auteurs se demandent si l'interprète est volontairement inclus dans les images, si l'on fait appel à lui ou à elle pour composer l'image, et qui assume la responsabilité de celle-ci. On peut ainsi se demander comment l'interprète est mis en scène (framed) ${ }^{8}$. Nous verrons que les jeunes interprètes gambadent autour de Reed, qu'ils somnolent ou s'agenouillent sous les yeux de Fisk, et même de Pope. Chez Smith, ils seront au côté du journaliste ou même devant lui. Même si cet aspect ne fait pas partie de notre analyse, se pose également la question des interventions éditoriales lorsqu'il s'agit d'éviter les mentions trop répétitives de la présence d'un médiateur linguistique et même celle de l'habitus du journaliste qui aurait assimilé le principe de l'invisibilité de l'interprète.

Nous tenterons donc d'analyser les images employées par les reporters pour décrire le travail des fixeurs et la manière dont la hiérarchie entre le reporter et le fixeur se manifeste, le cas échéant. Nous émettrons également en conclusion, après avoir présenté brièvement un contre-exemple, des hypothèses sur les raisons pour lesquelles, compte tenu de leur rôle primordial, ces médiateurs demeurent, malgré quelques apparitions ponctuelles, si invisibles.

\subsection{Corpus}

L'analyse se fonde sur le cas de quatre auteurs qui ne sont pas des reporters "parachutés", envoyés sur place pour produire un article puis disparaître aussitôt leur travail accompli (Wichtel 2004). Si ces derniers sont des experts des situations de crise, ils ne sont pas nécessairement experts de la région et de la crise concernées (Palmer et Fontan 1996: 100). À l'exception du premier, les auteurs analysés sont reconnus comme des experts de la région dont ils parlent.

- Robert Fisk, The Great War for Civilisation (2005).

o Né en 1946, ce Britannique vit au Liban depuis 1976 et parle arabe (les avis divergent sur son niveau de compétence). Il a été le correspondant de The Independent au Proche-Orient pendant plus de vingt ans. Fisk a publié un nombre important de livres sur la révolution iranienne, les guerres du Liban, du Golfe, d'Afghanistan ou l'invasion de l'Irak. Fisk retrace ici l'histoire du ProcheOrient à partir de ses expériences.

- Fred A. Reed, Anatolia Junction (1999).

o Journaliste et traducteur littéraire canadien né en 1939, il ne parle pas turc. Dans Anatolia Junction, il se met en quête du mouvement Nurculuk, fondé sur les idées de Saïd Nursi.

- Hugh Pope, Sons of the Conquerors, Dining with al-Qaeda (2010).

o Spécialiste du monde turc, de l'Asie centrale et de l'Iran, Pope a passé trente ans au Proche-Orient. Né au Cap en 1959, il a étudié le persan et l'arabe, et parle 
également turc. De 1997 à 2005, il a dirigé les bureaux du Wall Street Journal à Istanbul. Il est désormais directeur des communications et des relations extérieures de l'International Crisis Group.

- Graeme Smith, The Dogs Are Eating Them Now. Our War in Afghanistan (2013).

o Journaliste canadien né en 1979, il ne parle ni arabe, ni encore dari ou pachto, les deux principales langues de l'Afghanistan, mais vit à Kaboul où il supervise une équipe de l'International Crisis Group. Il a été correspondant pour le Globe and Mail (Toronto) à Kandahar, New Delhi et Istanbul.

Dans ces quatre cas, nous avons retenu les passages traitant spécifiquement de traduction ou d'interprétation. La recherche d'occurrences était relativement aisée dans les cas de Reed, Pope et Smith, compte tenu du volume des livres; l'analyse textuelle a permis de recenser les descriptions de situations de traduction ou d'interprétation chez Fisk.

\section{Mention des interprètes, des traducteurs et des fixeurs}

Encore une fois, l'objet n'est pas ici d'étudier le rôle ou la qualité du travail des interprètes-fixeurs, mais bien de s'interroger sur leur invisibilité fréquente dans les textes de nature journalistique et, le cas échéant, sur la manière dont ils sont représentés, évalués, mis en scène.

\subsection{Robert Fisk et la servilité des interprètes}

Fisk, arabophone, ne ressent pas le besoin d'évoquer ses intermédiaires éventuels, sauf lorsqu'il se trouve en Turquie; ce sont les autres qui traduisent, et il insiste sur les difficultés ainsi que sur les erreurs des interprètes, généralement des non-professionnels (fonctionnaires, militaires, etc.).

En 1980, en compagnie d'autres journalistes étrangers, il assiste à l'interrogatoire de deux prisonniers iraniens capturés par l'armée irakienne:

1) [T] he major stood round them in order to "translate." [...] "They are asking about your casualties," he said. The man shrugged and proclaimed his ignorance. "I am an Iranian soldier," he said quietly. Were the Iranian mullahs in charge of the Iranian army, journalists asked, and the major translated this question as: "Aren't religious people influencing your officers?" [...] And what, the world's press wanted to know, did the two prisoners think of Ayatollah Khomeini? The major mistranslated the question thus: "Now that things have gone so badly for you, what do you think of Khomeini?"

(Fisk 2005: 236)

Outre un cas d'interprétation non professionnelle, le passage présente des caractéristiques intéressantes. D'abord, la confusion des langues qui force le lecteur attentif à déconstruire la scène: au nom des journalistes étrangers (They), le major irakien et les représentants du gouvernement s'adressent au prisonnier iranien. Il faut supposer ici que l'un d'eux (lequel?) s'exprime en arabe et qu'un autre «interprète» traduit, ou encore qu'il parle persan (mais alors Fisk ne comprendrait pas et aurait donc besoin d'un interprète). Notons également dès le début les guillemets de l'auteur qui remettent en question le travail du major. L'Iranien répond ( $\underline{\mathrm{I} \text { am an Iranian }}$

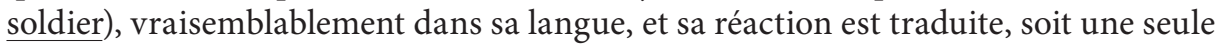


fois (en arabe), soit deux (en arabe puis en anglais). Les journalistes posent d'une seule voix la même question, probablement encore en anglais, mais rapportée en discours indirect: Were the Iranian mullahs in charge of the Iranian army? Question que le major traduit en persan par Aren't religious people influencing your officers? Les journalistes veulent encore savoir: What [...] did the two prisoners think of Ayatollah Khomeini? Traduction «erronée» du major: Now that things have gone so badly for you, what do you think of Khomeini?

L'ambiguité tient à l'absence de précision sur les langues, puisque l'on ne trouve ici aucune des incises usuelles (asked in English, replied in Arabic, etc.); rares dans les récits journalistiques, elles deviennent indispensables à la compréhension, sinon à la rigueur, lorsque plus de deux langues sont en présence. Fisk est ici l'autorité suprême, le narrateur omniscient d'une scène où au moins trois langues interagissent et qu'il maîtriserait prétendument toutes, mais la seule instance traductive évoquée est le major, interprète «inexact». Le journaliste commente ici, en anglais, les glissements de sens entre le persan et l'arabe ou entre l'anglais et le persan. Fisk ne parlant pas cette dernière langue, on peut se demander d'où il tient l'inexactitude de la traduction. De son propre fixeur?

La scène prend fin après l'ultime déclaration de l'un des prisonniers:

2) But the wounded man glanced quickly at us and said that "if Ayatollah Khomeini brought on a war between two Muslim countries, this was wrong." The conditional clause in this reply was lost on the Iraqi major who then happily ordered the removal of the prisoners?

(Fisk 2005: 236)

Le si (conditional clause) de la réplique anglaise, arabe ou persane échappe peutêtre au major, mais pas au journaliste, qui ne précise pourtant pas par combien de langues il est passé avant d'arriver à l'anglais.

Lorsqu'il n'est pas invisible, l'interprète pour Fisk semble une créature soit incompétente, soit vile et obséquieuse, ainsi que l'illustre le compte rendu de l'entrevue menée en 1979 avec l'ayatollah Khomeini, au moment de la crise des otages. Fisk est alors accompagné des journalistes américains John Hart et Peter Jennings, ainsi que de Sadeq Qotbzadeh, directeur de la télévision nationale et futur ministre des Affaires étrangères:

3) Qotbzadeh sat on Khomeini's right, gazing obsequiously at the man who would later condemn him to death, his head leaning towards the Ayatollah, anxious not to miss a single word. He, after all, would be the interpreter. So what of the embassy hostages? we wanted to know. Khomeini knew we would ask this. He understood the networks. His last, cynical remarks about newspapers in the final days of his life showed that he understood us journalists as well. [...] Of course, Hart and Jennings talked about international law, about the respect that should be paid to all embassies. The question was translated sotto voce by Qotbzadeh.

(Fisk 2005: 151-152)

L'interprète, Qotbzadeh, est ici nommé puisqu'il s'agit d'un politicien, d'une figure publique ${ }^{10}$. La scène prend fin par l'apparition soudaine d'un personnage absent jusque-là, soit l'interprète des journalistes (qui n'est pas Qotbzadeh). Là encore, l'interprétation est associée à la servilité: 
4) One of our drivers stepped forward - our own translator bent towards Khomeini and whispered that it would be the greatest moment in the driver's existence on earth if he could shake the Ayatollah's hand...

(Fisk 2005: 153)

La frontière est mince entre la posture attentive de l'interprète et celle du disciple soumis.

Ces deux cas sont des exceptions chez Fisk qui rend peu compte du rôle de la langue dans sa couverture du Proche-Orient. Encore une fois, dans les rares occasions où il mentionne la langue de l'interlocuteur, c'est qu'il s'agit d'une langue que Fisk ne parle pas (le russe, le turc) ou que son interlocuteur passe à une autre langue.

\subsection{Fred Reed ou la conscience de la traduction}

Le ton est tout autre chez Reed qui ne parle pas la langue du pays et qui laisse transparaître son amitié pour les fixeurs qui l'accompagnent dans son voyage initiatique en Anatolie. Ce sont ici des universitaires et des étudiants œuvrant, semble-t-il, à titre gracieux. L'auteur d'Anatolia Junction est celui qui décrit le plus en détail ses guides, tout en préservant parfois leur anonymat pour éviter les représailles, puisqu'il enquête sur un mouvement religieux plus ou moins licite en Turquie. Ils sont enthousiastes ou posés, selon leur âge, ou parfois les deux. Le jeune Erhan est avide de tout savoir: plies me with questions (Reed 1999: 92). Nurollah est un jeune élève appliqué: shy eighteen-year-old high school student, studious (Reed 1999: 94).

Certains compensent pour leur anglais défaillant par leur enthousiasme, tel le jeune Kemal, décrit comme un chiot frétillant:

5) Kemal, giggling compulsively, rolling his wide-set eyes and grinning with a slightly crooked mouth. In his excitement, the words came tumbling out, slipping and sliding over one another, in an effusion of stammering and lisping. Kemal reminded me of a bumbling, gamboling puppy.

(Reed 1999: 194)

Ou encore Murat, an English student who was my designated interpreter (Reed 1999: 234):

6) What he lacked in English skills, Murat more than compensated for in his eagerness to oblige. [...] We will meet in the morning for breakfast, Murat told me, repeating in the language of signs and gestures...

(Reed 1999: 234-235)

7) Please excuse me, Murat smiled, inserting a vowel between the two consecutive consonants to obtain the euphony required by Turkish...

(Reed 1999: 238)

Les autres interprètes désignés pour aider l'auteur dans sa quête sont des universitaires posés: Ferhat S., specialist in diseases of the nervous system [...] a physician of high repute (Reed 1999: 188); Hasan K., a member of the philosophy department, who took his graduate training in England (Reed 1999: 150); ou «Professor R.», a bespectacled lecturer at Istanbul University with a ready laugh and solid English (Reed 1999: 38-39). Reed se positionne en fait comme le sage qui effectue un voyage de découverte: les jeunes s'occupent de lui et les universitaires partagent avec lui leurs connaissances. 
Reed, traducteur lui-même, est celui qui est le plus attentif aux questions de traduction, mais sans juger, conscient qu'il est de sa propre ignorance de la langue locale et de la difficulté de la tâche. Deux cas sont ici pertinents, soit une description de «double interprétation» et l'entrevue avec le chef spirituel Fetullah Gülen.

Au monastère syriaque de Mardin, Reed recrute un interprète sur la base de deux critères: eagerness and ability to speak English (Reed 1999: 194). Il retient d'abord les services du jeune et timide Nurollah, mais se laisse convaincre d'adopter également Kemal:

8) But hovering at his elbow, another young man was shifting impatiently from one foot to the other. "Take me too" his eyes implored, before he blurted out: "I am from village [sic] near Mardin, I can help you." His infectious smile radiated enthusiasm; his name was Kemal. I could not refuse him.

(Reed 1999: 194)

Comme le bedeau (beadle) qui leur fait visiter le monastère parle le dialecte arabo-turc local, Kemal doit rendre ses propos une première fois en turc pour que Nurollah traduise en anglais :

9) His words must first pass through the filter of Kemal who, all atwitter, translates into Turkish for Nurollah, who then provides me with a twice-removed version. My questions follow the reverse order.

(Reed 1999: 98-99)

Dans le second cas, à Istanbul, Reed obtient finalement une audience avec Fetullah Gülen. L'entrevue est reconstruite, nous explique-t-il, à partir de l'enregistrement vidéo et de ses propres notes (Reed 1999: 81). Il pose ses questions par l'intermédiaire de l'interprète (vraisemblablement un employé de Gülen, qui reste invisible), mais le texte est donné en anglais sans mention d'un quelconque processus de médiation linguistique pendant plusieurs pages, jusqu'à la dernière partie du soliloque du chef spirituel:

10) "All countries should build bridges between each other, and open themselves to mutual investments and commercial activities. Schools opening all around the world would be very fruitful [...]." Such measures would lessen the danger of conflict. "They would fight the ignorant and arrogant 'Bedouin' reaction that we see in society," he says through the interpreter (though the phrase has disappeared from the transcript, as have the words "tribal ignorance" from a description of the South as poor, desolate and uneducated as compared to the cultured, sophisticated North). What did Mr. Gülen mean by "Bedouin" reaction, by "tribal ignorance"? Had he even uttered the words? Perhaps the frail craft of meaning had run aground on the shoals of simultaneous translation.

(Reed 1999: 86)

La mémoire de l'auteur lui ferait-elle défaut? Son interlocuteur a-t-il vraiment utilisé les expressions condescendantes « réaction de Bédouin» (Bedouin reaction) et «ignorance tribale» (tribal ignorance)? Le sens se serait-il échoué sur les récifs de l'interprétation simultanée? Le décalage entre les deux versions de l'interprétation correspond au trouble de Reed qui ressort «mal à l'aise» : ill-at-ease (Reed 1999: 86); peu importe le message de fraternité et de réconciliation, le laïus de Gülen reste trop opaque et ambigu. 


\subsection{Pope et le rêve d'avant Babel}

Malgré les différences de ton, Hugh Pope et Robert Fisk ont deux points en commun: ils explorent de nombreux pays (essentiellement du Proche-Orient et d'Asie centrale) et semblent communiquer le plus souvent sans difficulté avec leurs interlocuteurs, malgré la multitude de langues et de formes dialectales de l'arabe ainsi que des langues turciques. Pope, turcophone qui parle également arabe et persan, est donc le polyglotte de notre corpus, ce qui peut expliquer la rareté des mentions du travail d'un interprète. Chez lui, malgré la présence indéniable de fixeurs, que ce soit au Turkménistan, en Arabie saoudite ou en Iran, l'interprète, lorsqu'il est nécessaire ou même imposé, est vu avec méfiance. Pis encore, ironie du sort pour un métier qui est censé la faciliter, l'interprète est un obstacle à la communication, aussi laborieuse soit-elle, et même parfois un agent double (en Iran).

$\mathrm{Au}$ Turkménistan, l'opposant à la dictature que rencontre le journaliste insiste pour que celui-ci vienne seul (ce qui laisse entendre que la présence d'un interprète aurait été préférable):

11) He had insisted that I brought no interpreter. If Turkmen was relatively easy to read for someone who spoke Turkish, it seemed to be Central Asia's roughest, least-developed language when spoken. Uzbeks joke that the Turkmens speak as if they have a hot potato in their mouths. It is a spare, hard, secretive dialect, which evoked their unforgiving deserts, autocratic rulers, sudden violence, long periods of shortages and short moments of ecstatic plenty. We spoke slowly, he in Turkmen and I in Turkish, using up all our reserves of synonyms. [...] Still, we warmed in each other's company.

(Pope 2010: 99)

Malgré le caractère laborieux de la conversation et les risques liés à l'usage de «synonymes», mieux vaut se passer d'intermédiaire pour Pope et son interlocuteur qui préfèrent chercher les mots pour se comprendre.

La méfiance du Turkmène qui finit par s'ouvrir répond au récit des difficultés d'une historienne kazakhe avec laquelle Pope discute d'identité nationale:

12) [Klara] Sarabayeva spoke in Kazakh, and the translator was having trouble translating this high-minded talk into English. The Soviet Union had insisted that foreign languages only be learned through the medium of Russian. Sarabayeva tried that language on me, but it didn't help. I joked that if only we had a common knowledge of Turkish, things would be fine. Sarabayeva's mouth dropped open - she did speak Turkish! She had never been to Turkey, but had taught herself the kindred language and had come to love Turkish literature. As often happened, this discovery of a common Turkic medium filled the atmosphere with smiles and warmth. Sarabayeva sent her daughter out to bring more tea.

(Pope 2010: 183)

Bien sûr, traduire du kazakh vers l'anglais, soit probablement d'une langue $C$ vers la langue B, est une tâche malaisée pour un (une?) russophone, surtout lorsqu'il s'agit d'une conversation sérieuse (high-minded talk). Dans les deux cas, l'interprétation est tout de même un obstacle, soit par les limites de l'interprète, soit parce qu'elle constitue un risque. Peu importe le nombre de faux-amis entre le turkmène et le turc, mieux vaut se parler directement. Chaque fois, le turc est la lingua franca, le moyen de communiquer et de découvrir l'autre. Les deux scènes prennent fin par la rencontre 
chaleureuse des interlocuteurs: Still, we warmed in each other's company (exemple 11 ); this discovery of a common Turkic medium filled the atmosphere with smiles and warmth (exemple 12).

Pope le reconnaît volontiers, l'interprète nuit à la communication:

13) On some reporting trips I liked it when a colleague or a fixer helped me find my bearings or kept me company, but in places where I felt confident and spoke the language, like Saudi Arabia, I usually worked alone. It was easier to strike the necessary intense rapport with an interlocutor that way.

(Pope 2010: 139)

Le seul fixeur auquel Pope accorde une relative importance est celui qui l'accompagne dans sa découverte de la communauté yézidie, dans Dining with Al-Qaeda, Sagvan Murad, interprète kurde embauché à Dohuk. Murad a droit à plusieurs mentions au chapitre dix-sept, où il est décrit comme un young researcher (Pope 2010: 247) et un young and active member of an ancient religious community called the Yezidis (Pope 2010 : 272). On apprend également que le jeune homme fera carrière dans la fonction publique et deviendra chef du protocole du président d'Irak (Pope 2010: 285). C'est peut-être cette relative célébrité qui justifie la reproduction dans le livre d'une photo de Murad consultant un diseur de bonne aventure (Pope 2010: 276).

Soulignons-le, l'a priori contre l'interprétation (qui n'est pas sans rappeler l'objection préjudicielle contre la traduction) est à la fois celui des journalistes et de leurs interlocuteurs; dans les deux cas, les participants n'ont pas l'habitude de fonctionner en mode interprétation. Pope, point de départ de notre analyse de la représentation des fixeurs, est sans doute le plus romantique et le plus lettré des auteurs étudiés ici. Formé à Oxford en langues orientales, il cite abondamment les grands auteurs des pays qu'il visite, qu'il s'agisse de classiques ou de contemporains. Ses reportages sont truffés de références au canon littéraire persan ou turc. Les constantes allusions aux aventures d'Ármin Vambéry (1832-1913) sont un aveu: à l'instar de ce Hongrois, Pope rêve de se passer d'interprète. En Iran, les fixeurs sont parfois des agents des services secrets, raconte-t-il (Pope 2010 : 78), et en Chine, chez les Ouïghours, il faut employer un interprète pour obtenir un visa de journaliste (Pope 2005: 145).

L'une des rares représentations d'interprètes chez Pope montre des femmes accroupies dans les champs de tomates et de choux. Le journaliste assiste ce jour-là à des manœuvres militaires auxquelles participent Russes, Américains, Turcs, Kazakhs et Ouzbèkes, et décrit les difficultés de communication, lorsqu'il s'agit de se faire comprendre ou de lire les cartes:

14) There was no joint language between the Central Asians and their new friends from America. Indeed [...]. Translators from Central Asian ministries, mostly middle-aged ethnic Russian ladies, had been bundled up for the day in battle fatigues to help out. They crouched incongruously with American and Central Asian officers among the cabbage patches and tomato fields. Sign language turned out to be the easiest common medium.

(Pope 2005: 340)

Le ton cinglant de la description, le ridicule des interprètes accroupies dans les champs et décrites comme une masse impersonnelle deviendront dans le livre suivant la misère des fixeurs lorsque Pope évoquera ses débuts comme jeune journaliste à Damas, cherchant désespérément à frayer avec les grands reporters étrangers: 
15) These glamorous encounters made me hope that my lot as a twenty-two-year-old freelance fixer might one day become less lonely, monotonous, and impoverished.

(Pope 2010: 16)

\subsection{L'ange tutélaire de Graeme Smith}

Smith, le plus jeune de notre corpus, est, de son propre aveu, quelque peu dépassé par les événements à son arrivée en Afghanistan: ses interprètes seront donc souvent des anges gardiens. Le Canadien est également celui qui «représente» le plus les interprètes, que ce soit le sien ou ceux des autres.

Bien sûr, à plusieurs moments le lecteur pourrait croire que le journaliste s'adresse directement à ses interlocuteurs, par exemple, après le bombardement d'un village, lorsque les blessés se rendent au camp militaire pour se faire soigner. Smith s'adresse alors à un jeune garçon qui accompagne son oncle grièvement blessé:

16) [...] I asked him what happened. A bomb collapsed his house, he said. Four of his relatives were killed, and he could hear two of his younger brothers crying under the rubble. [...] When I asked him how he felt about the insurgents after the bombing, the boy looked at me seriously. His school had been closed for a year because the Taliban had beheaded four students.

(Smith 2013: 161)

$\underline{\text { I asked him signifie généralement we asked him ou plus précisément } \underline{I} \text { asked him }}$ through the interpreter, tout comme les passages au discours indirect.

Smith n'en est pas moins en rapport fusionnel avec son fixeur quasi maternel, prévoyant, sage, aguerri et rassurant. Le possessif systématiquement répété (my interpreter), utilisé pour éviter de désigner l'homme par son nom, crée également un sentiment de proximité, de complicité ainsi que le montrent quelques exemples où l'on voit l'interprète (Smith n'emploie jamais le terme fixer) se faire rassurant, protecteur ou prévoyant, en particulier dans les premières pages, où il explique au journaliste les règles de sécurité, lui fait visiter la région ou l'aide à prendre l'autocar:

17) My translator told me not to worry...

(Smith 2013: 15)

18) ... my translator woke me in the dimness before dawn.

(Smith 2013: 18)

19) ... my translator turned around in the front seat with a big grin. [...] My translator didn't seem worried about drawing attention to ourselves...

(Smith 2013: 19)

20) The only security advice from my translator was, "Mr. Graeme, please do not walk the streets at night." [...] My translator took me up the Forty Steps, an ancient stairway carved into a small mountain west of the city.

(Smith 2013: 20)

21) My translator shepherded me onto the old coach like a mentally impaired child and tucked me away in a window seat near the back. [...] When my translator recognized the landscape of Wardak province he visibly relaxed and informed me that Taliban were no longer a threat. [...] Some of the looks weren't welcoming, but my translator assured me that we had emerged from the danger zone...

(Smith 2013: 24) 
Le fixeur invisible ou incompétent que nous trouvions chez d'autres devient ici un être en chair et en os qui sourit, parle de sa propre voix, s'adresse directement à son interlocuteur, prend soin de lui. Le contraste est particulièrement frappant dans la scène où Smith se retrouve en présence d'un taliban et où l'interprète se transforme en garde du corps:

22) At one point he reached into his pocket and my translator lurched forward, preparing to throw himself at the fighter - this translator was useful in such situations, heavily muscled and trained in martial arts - but the insurgent only smiled and pulled out a metal box, not a weapon.

(Smith 2013: 95)

La situation est bien sûr beaucoup plus dangereuse que les scènes décrites par Reed ou Pope, mais pas que certaines racontées par Fisk. Lorsque l'interprète s'éloigne un moment, la tension monte d'un cran:

23) My translator left me alone with him for five minutes, and he started questioning me in rudimentary English: "Jesus or Moses?" He repeated the question over and over, with puzzling intensity, until my translator returned and assured him that I'm a Christian [...].

(Smith 2013: 95)

Lorsque Smith évoque les difficultés de communication, les interprètes ne sont pas coupables. Dans un cas, un jeune garçon s'évertue à traduire les arguments d'un général canadien à qui il essaie de faire comprendre l'inanité de ses efforts pour convaincre les policiers afghans de s'entraîner, mais peine à comprendre son anglais:

24) He had to repeat the question a couple of times because the translator had trouble with the soldier's French-Canadian accent. [...] Finally, the hard-faced policeman declared that he had more battlefield experience than any of the Canadians [...]. "I don't know what you could teach us," the [Afghan] policeman said. [...]

The Canadian gamely tried to continue with the lessons, until his interpreter decided it was silly to continue playing along.

"Sir, I don't think they care about these things," said the interpreter, a skinny kid in a blue baseball cap. [...]

The interpreter gamely made another pitch to the policemen, but only started a babble of argument.

"They're saying we've been doing this for years," the interpreter said. "I try to tell them, you suffer so many casualties, you must learn so you do not die."

"Good, good," the captain said. "Did they understand?"

"I think so," the interpreter said. But he looked uncertain.

(Smith 2013: 174-176)

Le dialogue a beau être rapporté en anglais, il est encadré par les mentions de l'interprète, au début et à la fin. De plus, nous avons cette fois un intervenant qui interprète littéralement le contexte au bénéfice de son "client», ajoutant à la simple traduction sa propre compréhension de la situation.

Dans un autre cas, un jeune Afghan est confronté au brouhaha de la conversation entre un militaire et des villageois qui s'expriment dans un dialecte difficile à saisir: 
25) "We know there are Taliban in this area but we don't see them," said the villagers through an interpreter, a teenager with the slightest fuzz of moustache on his upper lip. [...] "Since the government of Taliban changed they haven't seen any," the interpreter said, referring to the collapse of the previous regime in 2001. [...]

The interpreter struggled to keep up. Many of the young men hired as field translators were unmotivated, poorly paid considering the risks, and weren't familiar with local dialects of Pashto. It often seemed that only a small fraction of the words spoken in any of these meetings were understood by both parties. In this case, the interpreter gamely tried to convey the gist of the commander's words, but gave up when the villagers broke into a chorus of complaints. After a few minutes, he managed to summarize the elders' message...

(Smith 2013: 49)

Gamely (vaillamment) est le mot clé ici, qui décrit les deux jeunes interprètes, celui du général canadien-français et le suivant.

\subsection{Un contre-exemple: Le libraire de Kaboul}

La journaliste norvégienne Åsne Seierstad a été correspondante en Afghanistan. Dès l'introduction de son récit, Le libraire de Kaboul, Seierstad reconnaît n'avoir jamais appris le dari et avoir dû se fier aux membres de sa famille d'accueil qui parlaient anglais (Seierstad 2003: 12-13) ${ }^{11}$. Contrairement aux auteurs analysés ici, elle propose le portrait de Tajmir, un interprète improvisé qui se recycle lorsqu'un magazine américain lui offre un salaire qu'il ne peut refuser. À la différence de ce qui se passe chez Pope ou chez Smith, nous apprenons clairement comment les services du jeune garçon ont été retenus:

26) Il a été embauché comme interprète par un grand magazine américain. Avant, sous les talibans, il travaillait pour une organisation humanitaire. Il était responsable de la distribution de farine et de riz aux pauvres. [...] Lorsque les journalistes ont afflué à Kaboul, il a été sélectionné par ce magazine américain, où on lui proposait un salaire quotidien correspondant à ce qu'il percevait normalement pour quinze jours de travail. Il a pensé à sa propre famille, qui avait besoin d'argent, et a quitté son emploi pour devenir interprète, dans un anglais inventif et bizarre.

(Seierstad 2003: 301)

Le chapitre consacré à Tajmir lève le voile sur la routine du métier d'interprète, sur l'ennui qu'éprouve celui qui traduit toujours les mêmes questions, les sempiternelles réponses, et, surtout, qui ne rêve que de fuir les risques des expéditions pour retrouver sa femme:

27) Le seigneur de guerre se sert de la jambe de Tajmir pour souligner ses opinions, il dessine des cartes, des routes et des fronts sur sa cuisse. À chaque déclaration dont il est satisfait, il la claque avec force. Tajmir traduit comme un automate. Sur ses pieds grimpent les plus grosses fourmis qu'il ait jamais vues.

(Seierstad 2003: 310)

28) Tajmir traduit et traduit, les mêmes menaces, les mêmes propos. [...] En fait, il est assis là à traduire la raison pour laquelle la paix ne se fera jamais complètement en Afghanistan.

(Seierstad 2003: 319) 
Le récit ne se veut bien sûr pas un reportage, mais une forme de documentaire à la troisième personne. L'intérêt du portrait tracé par l'auteure est pourtant de présenter l'activité de Tajmir d'abord et avant tout comme celle d'un interprète et, ensuite, comme celle d'un intermédiaire ou d'un fixeur. Ajoutons que le portrait des personnages présenté par l'auteure est si proche de la réalité qu'il lui a valu des poursuites et des accusations.

Seierstad, contrairement aux quatre journalistes susmentionnés, est déjà en situation de traduction ou de diglossie puisqu'elle doit fonctionner en anglais et non en norvégien. Est-ce la raison pour laquelle elle est plus consciente des questions de langue et d'interprétation et prend soin de préciser dès l'introduction lesquels de ses interlocuteurs parlent anglais?

\section{Conclusion}

La visibilité du fixeur est plus grande chez Smith et Reed (même si celui-ci parle plutôt de "guides»). Ces deux Canadiens (citoyens d'un pays officiellement bilingue, seraient-ils plus sensibles à la rencontre des langues?) évoquent régulièrement le travail de leurs interprètes, de leurs chauffeurs et de leurs fixeurs, parfois une seule et même personne, même s'il faut souvent lire entre les lignes pour le comprendre. Chez Fisk, on ne trouve qu'une douzaine de mentions d'interprétation en quelque 800 pages et le journaliste n'évoque qu'en passant les cas où une forme de traduction est requise ou lorsque ce sont les autres qui en éprouvent le besoin. Il fait continuellement allusion à ses chauffeurs, mais jamais à un quelconque fixeur (on ne trouve aucune occurrence de l'expression). Pope, enfin, mentionne les interprètes le plus souvent in absentia ou lorsqu'ils défaillent, dans le cas de langues turciques trop éloignées du turc pour que la conversation soit aisée avec ses interlocuteurs. Le personnage clé des récits de Fisk et de Pope, pour qui l'on n'est jamais si bien servi que par soi-même, reste le chauffeur, beaucoup plus fiable que l'interprète.

Si les fixeurs décrits par ces quatre auteurs ont des points communs, leur représentation, elle, diffère de manière étonnante. Bien sûr, ce sont généralement des hommes, souvent de jeunes garçons (la langue anglaise permet cependant à Pope de laisser planer l'indéfini dans le cas du sexe des interprètes turkmène ou kazakh). Reed et Smith nous renseignent également quelque peu sur leur statut professionnel, du moins dans certains cas: universitaires, étudiants ou, parfois, journalistes. Lorsqu'il s'agit de relever les hésitations et les erreurs des interprètes, les quatre auteurs n'hésitent pas à décrire en détail la scène, qu'il s'agisse des militaires irakiens chez Fisk, d'une ou d'un interprète russophone chez Pope, de jeunes garçons à l'anglais hésitant chez Reed ou, enfin, chez Smith, de jeunes Afghans inexpérimentés. La compétence éthique et linguistique de ces interprètes varie: difficile d'établir une règle chez Pope et Fisk qui ne mentionnent la présence d'un intermédiaire linguistique que lorsque celui-ci défaille, mais Smith et Reed font preuve d'indulgence envers les jeunes garçons qui se sont improvisés interprètes.

Encore une fois, l'identité des divers fixeurs et interprètes reste indéfinie, soit pour leur sécurité, soit tout simplement parce que l'on ne les considère pas comme des personnages à part entière de la scène. Par définition, ils sont là parce que le journaliste ne parle pas la langue (et donc ne peut vérifier la qualité de l'interpréta- 
tion). Pourtant, Smith est à la fois celui qui se trouve dans le contexte le plus dangereux et celui qui accorde le plus de place à son interprète. Il est d'ailleurs le seul à rendre hommage aux interprètes dans ses remerciements (Smith 2013: 285).

Malgré les marques de gratitude, les fixeurs sont-ils vus comme des égaux capables de remettre en question la position du reporter et même de diverger d'opinion, ou ne sont-ils là que pour valider les perceptions du journaliste? On trouve ici deux types de représentation, soit d'abord celui de l'interprète comme un être humain, capable d'hésitations et de doutes, ou celui de l'interprète comme une machine qu'il est inutile de mentionner, un peu comme les écouteurs que l'on ne remarque qu'en cas de défaillance. La relative invisibilité des interprètes nous montre que certains journalistes, victimes, comme tant d'autres, de ce que l'on peut appeler le syndrome Star Trek qui implique l'existence d'un «traducteur universel», préfèrent nous faire oublier la barrière linguistique, à l'instar de tant de romans et de films de science-fiction où se posent rarement des problèmes langagiers. Tintin, le célèbre reporter, n'éprouve jamais de difficultés à communiquer avec les Syldaves, les Bordures ou les Américains, comme si tout le monde parlait français.

L'universalité de la communication chez Hergé (à de rares exceptions près, en français), qui entretient le mythe, passe bien sûr par l'invisibilité des intermédiaires, qu'ils soient traducteurs littéraires (ainsi que le montre bien Venuti) ou interprètes «non professionnels» (nos fixeurs). Si Venuti (1995: 5-6) s'intéresse essentiellement aux œuvres littéraires, ce qui rend son «invisibilité du traducteur» peu pertinente dans le cas qui nous intéresse, il n'en existe pas moins un point commun entre les deux contextes, soit le journaliste. Lorsqu'il est critique littéraire, ce dernier met encore souvent l'accent sur la transparence du texte et la clarté de la langue, sans pouvoir, ou vouloir, procéder à une véritable évaluation du texte de départ et de la traduction comme geste. Lorsqu'il est reporter, le journaliste, nos exemples le montrent, n'a le plus souvent pas les compétences linguistiques pour le faire. Malgré quelques exemples de commentaires sur le processus ou la qualité de l'interprétation dans notre corpus, là encore, l'analyse reste impressionniste. Dans les récits qui nous intéressent, l'invisibilité $d u$ fixeur apparaît (l'oxymore est volontaire) entre les lignes, dans les incises ou dans les commentaires. Contrairement à ce que connaît le plus souvent la traduction littéraire, nul n'a cependant accès aux échanges qui ont fait l'objet d'une interprétation; il n'y a donc pas (ou plus) d'«original». Paradoxalement, le «traducteur» a travaillé en présence de l'«auteur» du message et même du journaliste. Le modèle décrit par Venuti s'applique donc mal ici.

Dans les cas où les fixeurs entrent en scène, deux derniers éléments peuvent permettre de saisir le rôle que le journaliste leur assigne, soit leur position physique et leur représentation sous forme de clichés photographiques. La position du corps de l'interprète, nous l'avons vu chez Fisk et dans une moindre mesure chez Pope, est celle d'un être soumis, veule, ou simplement grotesque. Pope est tout de même le seul à proposer une photographie de l'un de ses fixeurs, celle de Murad consultant un diseur de bonne aventure yézidi. Chez Smith, on trouve une photo au début de chacun des chapitres, souvent de soldats canadiens ou afghans. La photo sur laquelle s'ouvre le chapitre 10, intitulée Afghan police in training, reluctantly (Smith 2013: 171), vraisemblablement prise pendant la formation décrite plus haut et à laquelle le jeune interprète participe, montre quatre policiers de plain-pied et deux autres, à moitié sur la photo; nulle trace du jeune interprète à la casquette bleue. Une ultime 
manière d'analyser le discours sur les fixeurs serait particulièrement intéressante dans le cas d'un corpus de langue anglaise, soit l'étude des verbes utilisés pour les décrire.

\section{NOTES}

1. VAmbéRY, Ármin (1886): Mes aventures et mes voyages dans l'Asie centrale. Paris: Alfred Mame et fils. 373 .

2. Witchel, Elisabeth (13 octobre 2004): The Fixers. Committee to Protect Journalists. Special Reports. Consulté le 30 juin 2019, <http://cpj.org/reports/2004/10/fixers.php>.

3. OfFice De LA LANGUe FRANÇAise DU Québec (24 septembre 2009): Le métier de fixer, ces anges gardiens des journalistes. OQLF. Pourquoi pas en français? Consulté le 15 août 2019, <https://web. archive.org/web/20180311225502/www.oqlf.gouv.qc.ca/actualites/capsules_hebdo/actualites_ terminolinguistique/pourquoi_fixer_20090924.html>.

4. Claude, Patrice (10 janvier 2005): Irak: l'avertissement de Jacques Chirac, le dilemme des médias. Le Monde. 2.

5. ORdRe Des traducteurs, terminologues et INTERPRÈtes du Québec (Dernière mise à jour: 5 novembre 2019): Aptitudes de l'interprète. OTTIAQ. Interprétation. Consulté le 17 décembre 2019, <https://ottiaq.org/a-propos/l-ordre/>.

6. "In any other sphere of professional interpreting activity, it is inadvisable to hire a young interpreter with no experience and no proper training. This is not the case in conflict interpreting, where the law of supply and demand rules - a "twelve year-old looking boy" can be hired as an interpreter» (Gómez Amich 2013: 17). Deborah Campbell est l'une des rares à décrire le travail d'une fixeur et, qui plus est, à lui donner un rôle central. Voir Campbell, Deborah (2014): A Disappearance in Damascus: A Story of Friendship and Survival in the Shadow of War. Toronto: Knopf Canada.

7. "Interpreters are often recruited because they "know" both the local language/dialect and English, the language of international relief operations, and not because they have been trained as translators or interpreters. It is safe to say that hardly any have undergone training in interpreting [...]» (s.p.). Voir Moser-Mercer, Barbara et Bali, Grégoire (3 juin 2008): Interpreting in zones of crisis and war. AIIC. Consulté le 30 juin 2018, <http://aiic.net/p/2979>.

8. "In the context of photography, the term designates a transformative effect that separates what is in the picture from what is not, creating visual and substantive relationships between the image's lines and shapes and its frame» (Shore 2007: 54-56, résumé et cité par Fernández-Ocampo et Wolf 2014: 1).

9. S’ajoute une difficulté supplémentaire, qui n’est pas évoquée dans le récit, soit la difficulté de comprendre l'arabe irakien dialectal, ainsi que le soulignent Palmer et Fontan, même pour les locuteurs natifs (Palmer et Fontan 2007: 8).

10. Fisk se rappelle l'homme plus tard et en parle encore en termes qui dénotent pour lui une soumission efféminée: «I remember the way Qotbzadeh talked to me about him, his voice softening into an almost feminine purr as he tried to convince me that the Ayatollah's annoyance at the slow pace of the revolution did not imply any change of character» (Fisk 2005: 154).

11. Puisqu'il s'agit d'une traduction du norvégien, nous avons choisi de ne pas inclure ici le texte original. Une première comparaison des passages évoqués ici montre cependant des différences significatives entre les versions française et anglaise.

\section{RÉFÉRENCES}

Apostolou, Fotini (2009): Mediation, manipulation, empowerment. Interpreting. 11(1):1-19.

Fernández-Ocampo, Anxo et Wolf, Michaela (2014): Framing the Interpreter: Towards a Visual Perspective. Londres/New York: Routledge.

Gómez Амісн, María (2013): The Vital Role of Conflict Interpreters. Nawa: Journal of Language \& Communication. 7(2):15-28.

LeAnZA, Yvan et Boivin, Isabelle (2008): Interpréter n'est pas traduire. Enjeux de pouvoir autour de l'interprétariat communautaire. In: Actes du colloque L'éducation en contextes pluriculturels: la recherche entre bilan et perspectives. (Colloque international «L'éducation en contextes 
pluriculturels: la recherche entre bilan et prospectives», Genève, 28-30 juin 2007). Consultée le $1^{\text {er }}$ décembre 2019, <https://www.researchgate.net/publication/242165999_Interpreter_n'est_ pas_traduire_Enjeux_de_pouvoir_autour_de_l'interpretariat_communautaire $>$.

Palmer, Jerry et Fontan, Victoria (2007): Our Ears and Our Eyes: Journalists and fixers in Iraq. Journalism. 8(1):5-24.

SHORE, Stephen (2007): The Nature of Photographs. Londres: Phaidon Press.

Venuti, Lawrence (1995): The Translator's Invisibility. Londres/New York: Routledge.

\section{ANNEXE: Références du corpus}

FISK, Robert (2005). The Great War For Civilisation: The Conquest of the Middle East, Londres: Fourth Estate. 236

Pope, Hugh (2005): Sons of the Conquerors: the Rise of the Turkic World. New York: Overlook Press.

Pope, Hugh (2010): Dining With Al-Qaeda: Three Decades Exploring the Many Worlds of the Middle East. New York: Thomas Dunne Books/St Martins Press.

ReEd, Fred A. (1999): Anatolia Junction: A Journey into Hidden Turkey. Vancouver: Talonbooks.

Seierstad, Åsne (2003): Le libraire de Kaboul. (Traduit par Céline Romand-Monnier). Paris: J.-C. Lattès.

Sмith, Graeme (2013): The Dogs Are Eating Them Now: Our War in Afghanistan. Toronto: Random House of Canada. 